Cahiers $d u$ MONDE RUSSE

\section{Cahiers du monde russe}

Russie - Empire russe - Union soviétique et États indépendants

50/2-3 | 2009

L'Europe orientale, 1650-1730. Crises, conflits et renouveau

\title{
Political Humor under StalinDavid BRANDENBERGER
}

, Bloomington : Slavica Publishers, 2009, 158 p.

\section{Gábor T. Rittersporn}

\section{(2) OpenEdition}

\section{Journals}

\section{Édition électronique}

URL : https://journals.openedition.org/monderusse/9762

DOI : 10.4000/monderusse. 9762

ISSN : $1777-5388$

\section{Éditeur}

Éditions de l'EHESS

\section{Édition imprimée}

Date de publication : 15 septembre 2009

ISBN : 978-2-7132-2260-3

ISSN : $1252-6576$

Référence électronique

Gábor T. Rittersporn, "Political Humor under StalinDavid BRANDENBERGER », Cahiers du monde russe [En ligne], 50/2-3 | 2009, mis en ligne le 14 janvier 2013, consulté le 03 septembre 2022. URL http://journals.openedition.org/monderusse/9762; DOI : https://doi.org/10.4000/monderusse.9762

Ce document a été généré automatiquement le 3 septembre 2022.

Tous droits réservés 


\section{Political Humor under StalinDavid BRANDENBERGER}

, Bloomington : Slavica Publishers, 2009, 158 p.

Gábor T. Rittersporn

\section{David BRANDENBERGER, Political Humor under Stalin, An Anthology of Unofficial Jokes and Anecdotes, Bloomington : Slavica Publishers, 2009, 158 p.}

1 Rien n'est plus compliqué que de raconter aux innocents des blagues soviétiques. Que de générations d'étudiants soviétiques et est-européens se sont évertuées, dans les foyers de Leningrad ou de Moscou, à faire passer le message aux camarades du Tiers-Monde ou d'Occident! Parfois l'échec était programmé. Car si le courant passait sans trop de problème, disons, entre Russes, Tchèques et Polonais, il fallait au Hongrois moyen environ deux mois pour saisir les chutes, ce qui était surtout dû au maigre effort qu'il avait investi au lycée dans ses études de russe. Restaient à expliquer, parfois même aux connaisseurs soviétiques, quelques noms qui ne figuraient guère dans les livres canoniques de l'histoire bolchevique. Par exemple, un Est-Européen risquait d'être mieux informé que beaucoup de Soviétiques au sujet de Radek, qui passait simplement pour un juif de plus dans la patrie de l'internationalisme prolétarien. Les amateurs se croyaient membres d'une société clandestine au savoir vaguement ésotérique. Quelle déception pour cette élite quand des citoyens exotiques commençaient à saisir sousentendus, allusions, ambiguïtés et autres nuances...

David Brandenberger ne tente donc pas l'impossible. Il publie environ trois cents blagues politiques publiées en 1951 à Munich par un certain Evgenii Andreevich. On ne sait rien sur ce quidam, pas même quand il a quitté l'URSS. Il se peut d'ailleurs qu'il s'agisse d'un pseudonyme. Brandenberger y ajoute une trentaine de blagues empruntées au « Harvard Project » - série d'entretiens réalisés à la même époque avec des réfugiés soviétiques, dans un anglais parfois hésitant. 
3 La plupart des blagues du recueil de Munich sont reproduites en russe et traduites en anglais. Parfois, l'ex-étudiant de Leningrad tire son chapeau. Souvent aussi, il se souvient des insurmontables difficultés qu'il rencontrait, même quand il traduisait dans sa langue maternelle; car bon nombre de blagues sont construites sur des jeux de mots qui n'ont pas d'équivalent dans d'autres langues. De plus, elles s'insèrent dans des contextes qui compliquent encore la tâche. Certes, un camarade malien, roumain ou argentin était suffisamment rodé au bout d'un certain temps pour comprendre et le russe et la culture soviétique. Mais les matheux ne communiquaient que par formules interposées. Du coup, leur connaissance du russe restait trop faible pour qu'ils en pénètrent toutes les subtilités, même lorsque le quotidien soviétique ne leur réservait plus de secrets.

On peut s'interroger sur l'acharnement de l'éditeur à publier cet ouvrage: les zélateurs des études russes n'auront aucun problème ; en revanche, le grand public y trouvera-t-il son compte? Pourtant, on soupçonne que Brandenberger s'adresse aussi à ce lectorat. D'où ses tentatives d'explication. Nous apprenons donc de Puškin qu'il était «noble, auteur et poète »; que Molotov était un "vieux bolchevik et hiérarque du parti ", Malenkov également. L'honnête homme ainsi renseigné butera peut-être sur le terme de "dékoulakisation», que l'éditeur ne déchiffre pas; cela dit, les historiens de la Russie ont tendance à abuser de ce néologisme : espérons seulement qu'il n'entrera pas au Grand dictionnaire d'oxford!

5 C'est sans doute à l'usage des profanes que l'éditeur destine son recueil d'illustrations. Pour la plupart, celles-ci sont tirées de deux livres de propagande. Ces dessins édifiants montrent des dirigeants, des ouvriers ou des paysans heureux dont les effigies n'ajoutent rien au texte. Sauf peut-être deux images dont Brandenberger n'a manifestement pas remarqué l'aspect anecdotique. Il se trouve qu'un dessinateur a réussi, la même année, à vendre un dessin identique pour une publication de propagande et pour un manuel d'histoire du parti qui faisait autorité à l'époque. L'artiste a seulement changé deux ou trois éléments pour transformer une paisible assemblée d'ouvriers en une réunion de purge du parti. Mais on ne doit pas trop en tenir rigueur à Brandenberger. L'éditeur de l'un de ces deux volumes, Sergej Ingulov, était le censeur qui allait devenir sous peu le chef de l'administration de la censure avant de tomber victime des purges pour avoir manqué de vigilance, certes pas dans le cas qui nous intéresse. Habent sua fata editori.

6 Brandenberger n'a décidément pas mérité le sort d'Ingulov. D'autant plus qu'il s'est donné une peine phénoménale pour repérer des variantes dans une multitude d'autres recueils et dans les matériaux du projet de Harvard, qu'il cite en notes. Il cite également des sources d'archives, des œuvres littéraires et fait référence à un grand éventail de textes qui vont des discours de Stalin jusqu'à des versets des Évangiles. Il lui arrive de se tromper, par exemple quand il pense que Mandel'štam maniait naïvement l'ironie dans l'épigramme sur Stalin qui lui a valu tant de souffrances. Le texte lui-même et ce que l'on sait de la biographie du poète suffisent à convaincre qu'il n'y avait aucune naïveté dans sa démarche. Mais ceci n'enlève rien à la valeur de l'ouvrage.

7 Le volume commence par passer en revue les recherches déjà effectuées sur la popularité des blagues soviétiques, leur public, les risques qu'encouraient ceux qui les faisaient circuler et le débat qui a sévi en URSS à la fin des années 1920 sur l'admissibilité de ce genre douteux de la satire dans une société qui prônait un avenir radieux. L'éditeur évoque également les problèmes de datation, mais ne s'y attarde peut-être pas suffisamment. C'est une aubaine que ses sources datent de l'immédiat après-guerre, mais ceci prouve seulement que les anecdotes du recueil étaient connues à cette date. Parfois, 
Brandenberger semble penser que des blagues qui portent, disons, sur la révolution ou la collectivisation, remontent à ces périodes : rien n'est moins sûr. Il est conscient que certaines anecdotes ont trouvé leur origine dans les milieux de l'émigration; néanmoins, il n'est pas suffisamment vigilant quand il évoque par exemple une histoire de hot-dog, objet certainement peu courant au pays des Soviets.

Le recueil est découpé en chapitres. Chacun porte sur une époque comme la NEP, l'industrialisation ou la guerre, et est précédé d'environ une page d'introduction. Là encore, les spécialistes n'apprendront rien et les lecteurs peu familiers de l'histoire soviétique resteront sur leur faim. Ajoutons que, très souvent, on voit mal sur quel critère telle blague a échoué dans tel chapitre. Enfin certaines anecdotes génériques passe-partout ne peuvent être associées à une époque précise.

Selon l'éditeur, ceux qui diffusaient des blagues politiques ne s'opposaient pas pour autant au régime. Il se serait agi de ridiculiser l'autorité et de contester les prétentions des bolcheviks. Sur ce point, Brandenberger a sans doute raison. Mais il n'est pas tout à fait sûr de son fait. Il fait remarquer que les blagues ont quelque chose en commun avec « la résistance limitée [et] inarticulée » décrite par James Scott comme une des armes du pauvre. Or, résistance implique opposition, même limitée.

De plus, dans les cas où les anecdotes politiques expriment la contestation, celle-ci est tout sauf inarticulée. Bien souvent, on y parle expressis verbis du désir de voir des dirigeants tués, y compris le Guide suprême, et de voir périr l'Union soviétique. Les farceurs n'étaient pas près de monter sur les barricades, mais ils ont indubitablement défié le régime et allaient jusqu'à contester sa légitimité. Il semblerait même qu'ils détruisaient symboliquement le pouvoir des Soviets. S'il est clair que ces blagueurs n'avaient aucune intention de passer à l'acte, néanmoins, ils faisaient preuve de dispositions d'esprit que des concepts comme «opposition» ou «résistance » ne rendent pas.

11 Certes, la plupart des blagues politiques ne véhiculaient pas une contestation radicale. Elles n'en donnent pas moins du fil à retordre aux chercheurs. Ceux-ci restent quelque peu prisonniers de schémas de pensée qui relèvent tantôt d'un militantisme digne d'un meilleur emploi, tantôt d'un pessimisme anthropologique (légèrement méprisant) au sujet d'un Homo sovieticus éternellement paralysé.

Du reste, l'anecdote, politique ou non, n'est pas un texte comme les autres. C'est un genre oral dont le message dépend beaucoup du contexte; de qui raconte, et à qui. Brandenberger montre que les plaisantins et leurs publics se recrutaient dans tous les milieux sociaux, des villages misérables jusqu'aux sommets du régime. Tout porte à penser que la même blague n'avait pas une signification tout à fait identique dans un foyer d'ouvriers, à la rédaction d'une revue, dans un camp de concentration et dans une soirée moscovite huppée. De même, l'historien de la Russie, qui l'interprétait d'une certaine manière dans les années 1970, le fait différemment aujourd'hui ; et sa lecture sera encore autre dans vingt ans. Il devrait donc largement nuancer ses savantes analyses et peut-être même se demander dans quelle mesure on peut parler de la blague soviétique en général.

13 Dès lors, il convient de manier celle-ci avec prudence. L'absence de certitude quant à sa datation fait qu'il est difficile de voir dans l'anecdote une réponse à un événement précis. La rareté des indications sur son environnement d'origine ne facilite pas la tâche d'exploration en tant que lieu de mémoire. C'est néanmoins une piste qui semble prometteuse. Une autre piste pourrait être celle des autorités et des tabous que les 
anecdotes remettent en question, déboulonnent ou cherchent à briser, ainsi que la nature des attaques. Les protagonistes, héros et vilains, et les stéréotypes sociaux et ethniques qui les caractérisent peuvent réserver des enseignements précieux. Il en va de même de leurs avatars dans diverses histoires et dans les variantes, mais une fois de plus, la datation risque de poser bien des problèmes. Amandine Regamey a publié un livre intéressant sur ce sujet, dont Brandenberger n'a malheureusement pas tiré les conséquences ${ }^{25}$.

14 L'éditeur pose la question de la comparaison avec des blagues politiques dans d'autres systèmes autoritaires, comme l'Allemagne nazie ou l'Espagne franquiste. Mais il serait aussi intéressant de comparer les anecdotes politiques et non politiques en Union soviétique. Les techniques rhétoriques, dialogiques et dramaturgiques, les situations, tabous, personnages, lieux et moments historiques révèlent sans doute des similarités et des contrastes qui peuvent faire apparaitre soit la particularité des anecdotes politiques, soit leur place à l'intérieur d'un genre qui ne permet peut-être pas facilement des découpages simplistes.

Les étudiants soviétiques et est-européens acceptaient mal l'idée que leur patrimoine humoristique, qu'ils croyaient si exceptionnel, soit accessible aux camarades n'ayant jamais vécu sous un régime communiste. C'est avec une condescendance à peine feinte qu'ils écoutaient des blagues venues des quatre coins du monde. Mais il y avait toujours un apprenti structuraliste pour rappeler des affinités et des analogies, même si les histoires n'avaient plus guère d'arrière-plan politique.

Et pour cause. Les blagues existent depuis la nuit des temps. Le plus vieux recueil connu date environ du troisième siècle avant notre ère et contient plus de deux cents histoires. Il a été compilé en Grèce (à laquelle nous avons emprunté le terme " anekdoton ») sous le titre évocateur de Philogelos, "l'amateur du rire». Mais il y a de bonnes raisons de soupçonner que cent cinquante ans plus tôt déjà, les comédies d'Aristophane faisaient référence à des blagues, y compris de nature politique. L'histoire d'Alexandre le Grand et de Diogène, ou celle de Vespasien et de l'argent qui n'a pas d'odeur, avaient déjà trait aux têtes couronnées. Et pourquoi ne pas aller plus loin, sans s'arrêter aux waraibanashi japonais, ces « contes à rire » qui ne sont qu'une variante d'un genre ancien qu'on risque de retrouver un peu partout.

Il serait important de replacer les blagues soviétiques dans ce contexte. L'effort exigerait la collaboration d'une vaste équipe internationale qui devrait commencer le travail par un répertoire des motifs, similaire à celui que nous avons pour les contes populaires. On ne pourrait qu'envier les chercheurs, car ils s'amuseraient beaucoup. Qui sait, peut-être aussi aux dépens des collègues qui persisteraient à clamer que les anecdotes soviétiques n'ont pas leur pareil au monde.

\section{NOTES}

25. Amandine Regamey, Prolétaires de tous pays, excusez-moi! Dérision et politique dans le monde soviétique, P. : Buchet-Chastel, 2007. 